\title{
Radial Multiresolution, Cuntz Algebras Representations and an Application to Fractals
}

\author{
Sergio Albeverio and Anna Maria Paolucci
}

\begin{abstract}
We study Bernoulli type convolution measures on attractor sets arising from iterated function systems on $\mathbf{R}$. In particular we examine orthogonality for Hankel frequencies in the Hilbert space of square integrable functions on the attractor coming from a radial multiresolution analysis on $\mathbf{R}^{3}$. A class of fractals emerges from a finite system of contractive affine mappings on the zeros of Bessel functions.We have then fractal measures on one hand and the geometry of radial wavelets on the other hand. More generally, multiresolutions serve as an operator theoretic framework for the study of such selfsimilar structures as wavelets, fractals, and recursive basis algorithms. The purpose of the present paper is to show that this can be done for a certain Bessel-Hankel transform.
\end{abstract}

Mathematics Subject Classification (2000). 42C40, 37G99, 43A32, 46E22, 43A62, 47C15.

Keywords. Hilbert spaces, Cuntz algebra, iterated function systems, radial multiresolution, Bessel functions, fractals.

\section{Introduction}

Multiresolutions were first envisioned in constructions of wavelet bases in $L^{2}(\mathbf{R})$, but as it turns out, they have uses in more general settings of operator theory. To see this, one may use certain representations of the Cuntz relations from operator algebra theory. In this paper this idea is applied to certain iterated function systems (IFSs). The analysis begins with an operator identity (the scaling law) stated for a system consisting of a single unitary operator which scales a particular IFS in a sense which is made precise in equations (1)-(2) below.

The starting point for the multiresolution analysis from wavelet theory is a system $U,\left\{T_{j}\right\}_{j \in \mathbf{Z}}$, of unitary operators with the property that the underlying complex Hilbert space $\mathcal{H}$ with norm $\|$.$\| , contains a vector \phi \in \mathcal{H},\|\phi\|=1$, 
satisfying

$$
U \phi=\sum_{j \in \mathbf{Z}} a_{j} T_{j} \phi
$$

for some sequence $\left\{a_{j}\right\}$ of complex scalars. In addition, the operator system $\left\{U, T_{j}\right\}$ must satisfy a non-trivial commutation relation. In the case of wavelets, it is

$$
U T_{j} U^{-1}=T_{N}, \quad j \in \mathbf{Z},
$$

where $N \in \mathbf{N}_{0}$ is the scaling number, or equivalently the number of subbands in the corresponding multiresolution. These relations play a role in signal processing and wavelet analysis. When this structure is present, there is a way to recover the spectral structure of the problem at hand from representations of an associated $C^{*}$-algebra. In the case of orthogonal wavelets, we may take this $C^{*}$-algebra to be the Cuntz algebra. In that case, the operators $T_{j}$ may be represented on $L^{2}(\mathbf{R})$ as translations,

$$
\left(T_{j} \xi\right)(x)=\xi(x-j), \quad \xi \in L^{2}(\mathbf{R}), \quad x \in \mathbf{R},
$$

and $U$ may be taken as the scaling $(U \xi)(x)=N^{-1 / 2} \xi(x / N), N \in \mathbf{N}$. This system clearly satisfies (2). (For a variety of other examples, the reader is referred to [18]. The setup there applies to dynamical systems of $N$-to-1 Borel measurable selfmaps: for example, those of complex dynamics and Julia sets.) In the wavelet case, a multiresolution is built from a $\phi \in L^{2}(\mathbf{R})$ satisfying (1). The numbers $\left\{a_{j}\right\}_{j \in \mathbf{Z}}$ from (1) must then satisfy the orthogonality relations

$$
\sum_{k \in \mathbf{Z}} a_{k}=1, \quad \sum_{k \in \mathbf{Z}} \bar{a}_{k} a_{k+2 m}=\delta_{0, m}, \quad m \in \mathbf{Z} .
$$

In this case, the analysis is based on the Fourier transform: define $m_{0}$ as a map from $S^{1}$ to $\mathbf{C}$ by

$$
m_{0}\left(e^{i t}\right)=\sum_{k \in \mathbf{Z}} a_{k} e^{i k t}, \quad t \in \mathbf{R} .
$$

Then (in the wavelet case, following [11]) a $\phi$ satisfying (1) in $\mathbf{H}=L^{2}(\mathbf{R})$ is given by the $L^{2}(\mathbf{R})$ product formula

$$
\hat{\phi}(t)=\prod_{j=1}^{\infty} m_{0}\left(t / N^{j}\right),
$$

up to a constant multiple ( $\hat{\phi}$ denoting the Fourier trasform of $\phi)$. The Cuntz algebra $O_{N}$ enters the picture as follows: formula (5) is not practical for computations, and the analysis of orthogonality relations is done better by reference to the Cuntz relations, see (11)-(12) below. Setting, for $\xi_{j} \in \mathbf{C}, j \in \mathbf{Z}$

$$
W\left(\left\{\xi_{j}\right\}\right):=\sum_{j \in \mathbf{Z}} \xi_{j} \phi(x-j),
$$


and using (3), we get an isometry $W$ of $\ell^{2}$ into a subspace of $L^{2}(\mathbf{R})$, the resolution subspace. Setting

$$
\left(S_{0} f\right)(z):=\sqrt{N} m_{0}(z) f\left(z^{N}\right), \quad f \in L^{2}(\mathbf{T}), \quad \text { Borel measurable, }
$$

$\mathbf{T}$ being the 1-torus and using the isomorphism $L^{2}(\mathbf{T}) \cong \ell^{2}$ given by the Fourier series, we establish the following crucial intertwining identity:

$$
W S_{0}=U W,
$$

so that $U$ is a unitary extension of the isometry $S_{0}$. It was shown in [3] and [4] that functions $m_{1}, \ldots, m_{N-1} \in L^{\infty}(\mathbf{T})$ may then be chosen such that the corresponding matrix

$$
\left(m_{j}\left(e^{i(t+k 2 \pi / N)}\right)\right)_{j, k=0}^{N-1}
$$

is in $\mathrm{U}_{N}(\mathbf{C})$ for (Lebesgue) a.a. $t \in \mathbf{R}$. Then it follows that the operators

$$
S_{j} f(z):=\sqrt{N} m_{j}(z) f\left(z^{N}\right), \quad f \in L^{2}(\mathbf{T}),
$$

will yield a representation of the Cuntz relations; see (11)-(12) below. Conversely, if (10) is given to satisfy the Cuntz relations, then the matrix in (9) takes values in $\mathrm{U}_{N}(\mathbf{C})$. The scaling could be between different resolutions in a sequence of closed subspaces of the underlying Hilbert space or it could refer to a system of frequency bands. Two structures are then present: a scaling from one band to the next and operations within each band. These two operations can be realized in a certain tensor factorization of the Hilbert space.

The present paper aims at a generalized construction in which the two operations are realized in a radial setting. The system will be based on the Bessel functions, i.e., we use the Bessel functions with a given (real) order $\alpha$ in (4) in place of the usual Fourier basis $\left\{e^{i k t}\right\}_{k \in \mathbf{Z}}$. The Fourier transform will then be replaced by Hankel transforms. Our use of the Cuntz algebra is motivated by [3] and [5]. The Cuntz algebras [10] have been used independently in operator algebra theory and in the study of multiresolution wavelets, and our present paper aims to make this connection explicit, and, at the same time, to extend the analysis to the case of a radial scaling. A key link between wavelets and fractals on the analysis side and random walks on the probability side is to be found in the use of the filters from signal processing. The study of the filter functions is part of signal processing. They are one of the most useful tools for the construction of wavelets and also of wavelets applications. In our context, an infinite matrix product involving the matrix $W=m^{\star} m$, constructed from filter functions, create a positive path measure of a random walk starting at $x$ in some given period interval.The approach to the analysis of signals given by the corresponding wavelets may be understood via a random walk model, namely a random walk on a combinatorial tree with a suitable $N$-fold branching. Using such models we construct a class of infinite products which are connnected to random walks and arise in a special case from radial wavelets. 


\section{Cuntz algebras and iterated function systems}

We recall that one denotes by $O_{N}$ the $C^{*}$-algebra generated by $N, N \in \mathbf{N}$, isometries $S_{0}, \ldots, S_{N}$ satisfying

$$
S_{i}^{*} S_{j}=\delta_{i j} \mathbf{1}
$$

and

$$
\sum_{i=0}^{N} S_{i} S_{i}^{*}=\mathbf{1} .
$$

where $i, j=1, \ldots, N$. The representations we will consider are realized on Hilbert spaces $H=L^{2}(X, \nu)$, where $X$ is a measure space which will be specified later and $\nu$ is a probability measure on $X$.

We define the representations in terms of certain maps

$$
\begin{aligned}
\sigma_{i}: \Omega \longrightarrow \Omega \text { such that } \Omega= & \bigcup_{i=0}^{N-1} \sigma_{i}(\Omega) \\
& \text { and }\left(\sigma_{i}(\Omega) \cap \sigma_{j}(\Omega)\right)=0 \text { for all } i \neq j .
\end{aligned}
$$

We identify a class of representations of the Cuntz algebra based on a fractal measure space.

Let us recall that the Hankel transform of order $\alpha$ of a function $f$, denoted by $\hat{f}$, is defined by

$$
\hat{f}(t)=\int_{0}^{\infty} J_{\alpha}(x t) f(x) x d x
$$

where

$$
J_{\alpha}(x)=\left(\frac{x}{2}\right)^{\alpha} \sum_{k=0}^{\infty} \frac{(-1)^{k}}{k ! \Gamma(\alpha+k+1)}\left(\frac{x}{2}\right)^{2 k}
$$

is the Bessel function of order $\alpha, \alpha \in \mathbf{R}$ and $\Gamma$ is the classical gamma function, i.e.,

$$
\Gamma(z)=\int_{0}^{\infty} e^{-t} t^{z-1} d t, \quad \operatorname{Re}(z)>0 .
$$

If we multiply both sides of (14) by $J_{\alpha}(y t) t$ and integrate from $t=0$ to $+\infty$ we obtain, for Borel measurable functions $f$ such that all integrals exist:

$$
\int_{0}^{\infty} J_{\alpha}(y t) \hat{f}(t) t d t=f(y)=\int_{0}^{\infty} J_{\alpha}(y t) t\left(\int_{0}^{\infty} J_{\alpha}(x t) f(x) x d x\right) d t .
$$

This is the Hankel inversion theorem, see, e.g., [31]. The resulting double integral is called the Hankel Fourier-Bessel integral

$$
f(y)=\int_{0}^{\infty} J_{\alpha}(y t)\left(\int_{0}^{\infty} J_{\alpha}(x t) f(x) x d x\right) t d t .
$$


It can be written in terms of the following transform pair

$$
\begin{aligned}
& g(t)=\int_{0}^{\infty} J_{\alpha}(y t) f(y) y d y, \\
& f(y)=\int_{0}^{\infty} J_{\alpha}(y t) g(t) t d t .
\end{aligned}
$$

A Plancherel type result can easily be derived for this transform: if $F(\rho)$ and $G(\rho)$ are the Hankel transforms of $f(x)$ and $g(x)$ respectively, then we have

$$
\begin{aligned}
\int_{0}^{\infty} \rho F(\rho) G(\rho) d \rho & =\int_{0}^{\infty} \rho F(\rho) \int_{0}^{\infty} x g(x) J_{\nu}(\rho x) d x d \rho \\
& =\int_{0}^{\infty} x g(x)\left(\int_{0}^{\infty} \rho F(\rho) J_{\nu}(\rho x) d \rho\right) d x=\int_{0}^{\infty} x f(x) g(x) d x .
\end{aligned}
$$

As mentioned in the Introduction, it is our aim here to use a generalized construction of multiresolutions based on a radial scaling to construct classes of measures via representations of the Cuntz algebras. To make that explicit let us recall a few definitions we need about radial multiresolution.

Suppose that $F \in L^{2}\left(\mathbf{R}^{\mathbf{d}}\right)$ is radial, i.e., $F(A x)=F(x)$ for all $A \in S O(d)$. Then there is a unique $f \in L^{2}\left(\mathbf{R}_{+}, \omega_{\mathbf{d} / \mathbf{2}-\mathbf{1}}\right)$ such that $F(x)=f(|x|)$, where the |.| denotes the Euclidean norm on $R^{d}$ and for $\alpha \geq-1 / 2$, the measure $\omega_{\alpha}$ on $R^{+}=[0, \infty)$ is given as before by

$$
d \omega_{\alpha}(r)=\left(2^{\alpha} \Gamma(\alpha+1)\right)^{-1} r^{2 \alpha+1} d r .
$$

Its normalization implies that $\|F\|_{2}=\|f\|_{2, \omega_{d / 2-1}}$, where $\|\cdot\|_{2}$ is taken with respect to the normalized Lebegue measure $(2 \pi)^{-d / 2} d x$ on $R^{d}$.

On $L^{2}\left(\mathbf{R}_{+}, \omega_{\mathbf{d} / \mathbf{2}-\mathbf{1}}\right)$ the Hankel transform of index $\alpha$ is given by

$$
\hat{f}(t)=\int_{0}^{\infty} J_{\alpha}(x t) f(x) d \omega_{\alpha}(r),
$$

where

$$
J_{\alpha}(x)=\left(\frac{x}{2}\right)^{\alpha} \sum_{k=0}^{\infty} \frac{(-1)^{k}}{k ! \Gamma(\alpha+k+1)}\left(\frac{x}{2}\right)^{2 k}
$$

is the Bessel function of order $\alpha, \alpha \in \mathbf{R}$. Let $H$ be the Hilbert space $L^{2}\left(\mathbf{R}_{+}, \omega_{(\alpha)}\right)$, where the measure $\omega_{(\alpha)}$ on $R_{+}$is given by (18) with $\alpha \geq-1 / 2$. The pair $H_{\alpha}=$ $\left(\mathbf{R}_{+}, \omega_{(\alpha)}\right)$ is called the Bessel-Kingman hypergroup of index $\alpha$, see, [32]. Let $Q$ be a countable subset of $(0, \infty)$ and let $g \in L^{2}\left(H_{\alpha}\right)$. We assume that the support of $\hat{g}$ is contained in $[0, l]$ for some $l \geq 0$, where $\hat{g}^{\alpha}$ is the Hankel transform of index $\alpha$, i.e.,

$$
\hat{g}^{\alpha}(\lambda):=\int_{0}^{\infty} j_{\alpha}(\lambda r) f(r) d \omega_{(\alpha)} .
$$

We will consider the Hankel transform using the following normalized Bessel function

$$
j_{\alpha}(x)=\Gamma(\alpha+1)(z / 2)^{-\alpha} J_{\alpha}(x) .
$$


There is a Plancherel theorem for the Hankel transform, which states that

$$
f \rightarrow \hat{f}^{\alpha}
$$

establishes a self-inverse, isometric isomorphism of $L^{2}\left(\mathbf{R}_{+}, \omega_{\mathbf{d} / \mathbf{2}-\mathbf{1}}\right)$, (see, e.g., [29]). If $F \in L^{2}\left(\mathbf{R}^{\mathbf{d}}\right)$ is radial with $F(x)=f(|x|)$, then its Plancherel transform

$$
\mathcal{F}(F)(\xi)=(2 \pi)^{d / 2} \int_{\mathbf{R}^{\mathbf{d}}} F(x) \exp (-i\langle x, \xi\rangle) d x
$$

is again radial with $\mathcal{F}(F)(\xi)=f^{d \hat{2}-1}(|\xi|)$. We also observe that the spherical means

$$
M_{r} F(x):=\int_{S^{d-1}} F(x+r \xi) d \sigma(\xi)
$$

with $r \in \mathbf{R}_{+}$, of $F$ are again radial. We have $\left\|M_{r} F\right\|_{2} \leq\|F\|_{2}$, thus $M_{r}$ induces a norm-decreasing linear mapping

$$
T_{r}: L^{2}\left(\mathbf{R}_{+}, \omega_{\mathbf{d} / \mathbf{2}-\mathbf{1}}\right) \rightarrow L^{2}\left(\mathbf{R}_{+}, \omega_{\mathbf{d} / \mathbf{2}-\mathbf{1}}\right)
$$

and $T_{r} f(|x|):=M_{r} F(x)$ where $F$ and $f$ are related as above. Put $\alpha=d / 2-1$, then by using polar coordinates we get:

$$
T_{r} f(s):=c_{\alpha} \int_{0}^{\pi} f \sqrt{\left(r^{2}+s^{2}-2 r s \cos \phi\right)} \sin ^{2 \alpha} \phi d \phi,
$$

with $c_{\alpha}=\Gamma(\alpha+1) / \Gamma(\alpha+1 / 2) \Gamma(1 / 2)$. This defines a norm-decreasing generalized translation on $L^{2}\left(\mathbf{R}_{+}, \omega_{\mathbf{d} / \mathbf{2}-\mathbf{1}}\right)$, not only for $\alpha=d / 2-1$ but also for general $\alpha \geq-1 / 2$.

Let us observe that for the generalized translation the following property holds:

$$
\int_{0}^{\infty}\left(T_{s} f\right) g d \omega_{\alpha}=\int_{0}^{\infty} f\left(T_{s} g\right) d \omega_{\alpha},
$$

for $s \in \mathbf{R}_{+}$, whenever both integrals exist. Thus the Hankel transform $f \rightarrow \hat{f}^{\alpha}$ can be interpreted as a Plancherel transform for the hypergroup $H_{\alpha}$ on $L^{2}\left(H_{\alpha}\right):=$ $L^{2}\left(\mathbf{R}_{+}, \omega_{\alpha}\right)$. Hence from the above property we get the following:

$$
\left(\hat{T}_{r} \hat{f}^{\alpha}\right)(\lambda)=j_{\alpha}(\lambda r) \hat{f}^{\alpha}(\lambda) .
$$

Now we want to turn to the case where $N=3$ which corresponds to $\alpha=\frac{1}{2}$. The radial multiresolution in $\mathbf{R}^{3}$ is a construction of a wavelet-type orthonormal basis for the space of radial $L^{2}$ functions in $\mathbf{R}^{3}$. The elements of the basis are obtained from a single radial wavelet by usual dilations and generalized translations. The generalized translation reveals the group convolution of radial functions in $\mathbf{R}^{3}$. By Lemma 4.10 in [30], given $\phi \in L^{2}(\mathbf{H})$ as an orthonormal scaling function of a radial multiresolution, the associated filter function $G$ satisfies

$$
|G(\lambda)|^{2}+|G(\lambda+1)|^{2}=1 \quad \text { a.e. }\left(\omega_{\alpha}\right),
$$


with $\left(\omega_{\alpha}\right)$ defined in $(18)$. Hence $G$ is essentially bounded and contained in $L^{2}([0,1])$, which allows us to develop it into a cosine series

$$
G(\lambda)=\sqrt{2} \sum_{n=0}^{\infty} g_{n} \cos (n \pi \lambda),
$$

which converges in $L^{2}(0,1)$.

Then the infinite product

$$
\prod_{j=1}^{\infty} G\left(3^{-j} \lambda\right)
$$

is the Fourier transform of an infinite convolution measure $\mu$ such that:

$$
\widehat{\mu}=\left(\prod_{j}\left(\sum g_{n} \cos \left(n \pi 3^{-j} \lambda\right)\right)\right) .
$$

The convergence of the infinite product in (23) follows from Theorem 8.11 in [15].

Now, let us consider the Cuntz algebra relations, (11)-(12). The study of these relations involves a detailed analysis of some unitary operator in a Hilbert space $H$ which plays the role of scaling. Let $S_{i} \in \operatorname{Rep}\left(O_{N}, H\right)$ be a system of operators in the Hilbert space $H$.

We consider a permutative representation of $O_{N}$ in the sense of [5]. By Lemma 9.3.5 [5] every permutative representation of $O_{N}$ on a Hilbert space $H$ has the following form:

$$
S_{i}|x\rangle:=\left|\sigma_{i}(x)\right\rangle, \quad x \in X
$$

for some set $X$ and an $N$ - fold branch mapping $\sigma: X \rightarrow X,[19]$, where $|x\rangle,\left|\sigma_{i}(x)\right\rangle$ are vectors in $H$ (Dirac notation).

Thus

$$
S_{i}^{*}\left|\sigma_{i}(x)\right\rangle:=\delta_{i, j}|x\rangle
$$

with $i, j=1, \ldots, N$.

The maps $\sigma_{i}$ are one-to-one and satisfy for $x \in X$.

$$
\sigma_{i}(X) \bigcap \sigma_{j}(X)=\emptyset
$$

if $i \neq j$ and

for $i=1, \ldots, N$ and $x \in X$.

$$
\bigcup_{i=1}^{N} \sigma_{i}(X)=X
$$

The isometries generating $O_{N}$ provide subdivisions of the Hilbert space $H$ in view of

and

$$
S_{i}^{*} S_{j}=\delta_{i j} \mathbf{1}
$$

$$
\sum_{i=0}^{N} S_{i} S_{i}^{*}=1
$$


In particular for every $k \in \mathbf{N}$ the subspaces :

$$
H\left(a_{1}, a_{2}, \ldots, a_{k}\right):=V_{a_{1}} V_{a_{2}} \ldots V_{a_{k}} H
$$

are mutually orthogonal and one has

$$
\sum_{i_{1}, i_{2}, \ldots, i_{k}} H\left(a_{1}, a_{2}, \ldots, a_{k}\right)=H .
$$

If $f \in H$ and $\|f\|=1$ then

$$
\mu_{f}(.):=\langle f, E(.) f\rangle=\|E(.) f\|^{2}
$$

is a probability measure on the unit interval $[0,1]$. We want to specialize $E($.$) to$ our case and compute the associated measure. Let us observe that the index labels $\left(a_{1}, a_{2}, \ldots, a_{k}\right)$ are used to assign $N$-adic partitions (e.g., the intervals $\left[\frac{a_{1}}{N}+\cdots+\right.$ $\left.\left.\frac{a_{k}}{N^{k}}, \frac{a_{1}}{N}+\cdots+\frac{a_{k}}{N^{k}}+\frac{1}{N^{k}}\right]\right)$, then we have the mapping

$$
\left(a_{1}, \ldots, a_{k}\right) \rightarrow H\left(a_{1}, a_{2}, \ldots, a_{k}\right),
$$

where $\left(a_{1}, a_{2}, \ldots, a_{k}\right) \in\{(0,1, \ldots, N)\}$ and the length of the interval is $\frac{1}{N^{k}}$. These partitions are a special case of endomorphisms

$$
\sigma: X \rightarrow X,
$$

where $X$ is a compact Hausdorff space and $\sigma$ is continuous and onto. For every $x \in X$ we have that $\operatorname{card}\left(\sigma^{-1}(x)\right)=\{x \in X / \sigma(y)=x\}=N$. There exists branches of the inverse, i.e., maps

$$
\sigma_{0}, \ldots, \sigma_{N-1}: X \rightarrow X
$$

such that

$$
\sigma \circ \sigma_{i}=1_{X} .
$$

For each $0 \leq i<N$ the above intervals written in terms of the maps $\sigma_{i}$ are:

$$
\begin{aligned}
I_{k}(a) & =\left[\frac{a_{1}}{N}+\frac{a_{2}}{N^{2}}+\cdots+\frac{a_{k}}{N^{k}}, \frac{a_{1}}{N}+\frac{a_{2}}{N^{2}}+\cdots+\frac{a_{k}}{N^{k}}+\frac{1}{N^{k}}\right] \\
& =\sigma_{a_{1}} \circ \sigma_{a_{2}} \cdots \circ \sigma_{a_{k}}(X) .
\end{aligned}
$$

The system $\sigma_{a}=\sigma_{a_{1}} \circ \sigma_{a_{2}} \ldots \circ \sigma_{a_{k}}$ forms a set of branches for $\sigma^{k}=\sigma \circ \sigma \ldots \circ \sigma$ and is called an $N$-adic system of partitions of $X$. Thus, for every $k \in \mathbf{Z}_{+},\left\{J_{k}(a)\right\}$ is a partition indexed by $a \in \Gamma_{N}^{k}, \Gamma_{N}^{k}:=\Gamma_{N} \times \Gamma_{N} \times \cdots \times \Gamma_{N}$. On the other hand, given a Hilbert space $H$, a partition of projections in $H$ is a system $P(i)_{i \in I}$ of projections, i.e., $P(i)=P(i)^{*}=P(i)^{2}$, such that

$$
P(i) P(j)=0
$$

if $i \neq j$ and

$$
\sum_{i \in \mathbf{I}} P(i)=1_{H} .
$$

Let $N \in \mathbf{N}, N \geq 2$. Suppose that for every $k \in \mathbf{N}$, there is a partition of projections $P_{k}(a)_{a \in \Gamma_{N}^{k}}$ such that every $P_{k+1}(a)$ is contained in some $P_{k+1}(b)$, i.e., $P_{k}(b) P_{k+1}(a)=P_{k+1}(a)$, then $P_{k}(a)_{a \in \Gamma_{N}^{k}}$ is a system of partitions of $1_{H}$. By 
Lemma 3.5 [24] given an $N$-adic system of projections of $X$ and $\left\{P_{k}(a)\right\}_{k \in \mathbf{Z}_{+}, a \in \Gamma_{N}^{k}}$ an $N$-adic system of projections, there is a unique normalized orthogonal projection-valued measure $E($.$) defined on the Borel subsets of X$ with values in the orthogonal projections of $H$ such that $E\left(J_{k} a\right)=P_{k}(a)$ for every $k \in \mathbf{Z}_{+}, a \in \Gamma_{N}^{k}$. Let $S_{i}$ be the generators of the Cuntz algebra $O_{N}$ represented on a Hilbert space $H$. Assume $a=\left(a_{1}, a_{2}, \ldots, a_{k}\right) \in \Gamma_{N}^{k}$ and set $S_{a}:=S_{\left(a_{1}\right)} \ldots S_{\left(a_{k}\right)}$, then $P_{k}(a)=S_{(a)} S_{(a)}^{*}$.

Thus:

$$
\begin{aligned}
\mu_{f}\left(I_{k}(a)\right) & =\left|E\left(I_{k}(a)\right)\right|^{2}=\left\|S_{(a)} S_{(a)}^{\star} f\right\|^{2} \\
& =\left\langle f, S_{(a)} S_{(a)}^{\star} f\right\rangle \\
& =\left\|S_{(a)}^{\star} f\right\|^{2} \\
& =\sum_{n \in \mathbf{Z}}\left|\left\langle e_{n}, S_{(a)}^{*} f\right\rangle\right|^{2} \\
& =\sum_{n \in \mathbf{Z}_{+}}\left|\left\langle S_{(a)} e_{n}, f\right\rangle\right|^{2} .
\end{aligned}
$$

The following result characterizes the above projection-valued measure induced from a given representation of the Cuntz algebra $O_{N}$.

For the rest of the section we assume $N=3$.

Theorem 1. Let $\left(S_{k}\right)$ be of the form:

$$
S_{k} f(z):=\sqrt{3} m_{k}(z) f(\sigma(z)), \quad f \in L^{2}(\mathbf{T}), \quad \text { with } \quad\|f\|=1
$$

where $m_{k}(z)=j_{\alpha}\left(z r_{k} \exp \left(i 2 \pi \frac{k}{3}\right)\right), r_{k}$ are the zeros of the normalized Bessel function given by $(20) j_{\alpha}$ in $[0,1], k \in \mathbf{Z}_{+}, a=\left(a_{1}, \ldots, a_{k}\right) \in \Gamma_{3}^{k}$, with $\Gamma_{3}^{k}$ defined by $\Gamma_{3} \times \Gamma_{3} \times \cdots \times \Gamma_{3}$. Then the operators $\left(S_{k}\right)$ give a representation of the Cuntz algebra $\mathrm{O}_{3}$ on a Hilbert space $H$. The measure $\mu_{f}$ induced from the operators $\left(S_{k}\right)$ is given by the formula:

$$
\mu_{f}\left(I_{k}(a)\right)=\sum_{n \in \mathbf{Z}}\left|\prod_{i=0, \ldots k-1}\left(T_{r_{i}} f\right)^{\wedge}\left(n 3^{i}\right)\right|
$$

where $T_{r}$ is defined by (21), and ${ }^{\wedge}$ is the Hankel transform given by (22).

Proof. The operators $S_{k}$ have the following form:

$$
S_{k}\left|z^{i}\right\rangle=j_{(\alpha)}(z \exp (i 2 \pi k / 3))\left|z^{3 i}\right\rangle
$$

then the adjoint operator $S_{h}^{*}$ is such that

$$
\begin{aligned}
S_{h}^{*} S_{k}\left|z^{i}\right\rangle & =\left\langle j_{(\alpha)}\left(z r_{h}(\exp (i 2 \pi h / 3))\right), j_{(\alpha)}\left(z r_{k}(\exp (i 2 \pi k / 3))\right\rangle\left|\sigma_{h}\left(z^{3 i}\right)\right|\right\rangle \\
& =\delta_{h, k}\left|z^{i}\right\rangle .
\end{aligned}
$$

By using the properties of the Bessel functions with respect to their zeros we easily get that the Cuntz relations are satisfied, (11)-(12). For this is useful to note that 
if $r_{n}$ is a zero of the Bessel function $j_{\alpha}$ then also $\exp (i 2 \pi k / 3) r_{n}$ is a zero of the same function. By using the properties of the Bessel functions and the operators $S_{k}$ defined above we get for $k=1$ :

$$
\begin{aligned}
\mu_{f}\left(I_{1}(a)\right) & =\sum_{n \in \mathbf{Z}}\left|\left\langle j_{\alpha}\left(z r_{1} \exp \left(2 i \pi \frac{a_{1}}{3}\right)\right) e_{n}\left(z^{3}\right), f\right\rangle\right|^{2} \\
& =B\left(a_{1}\right) \sum_{n \in \mathbf{Z}}\left|\left\langle j_{\alpha}\left(z r_{1}\right) e_{n}\left(z^{3}\right), f\right\rangle\right|^{2} \\
& =B\left(a_{1}\right) \sum_{n \in \mathbf{Z}}\left|\widehat{f e_{n}(n 3)}\right|^{2}=B\left(a_{1}\right) \sum_{n \in \mathbf{Z}}\left|\left(T_{r_{1}}\left(\bar{f} e_{n}\right)\right)^{-}(3)\right|^{2}
\end{aligned}
$$

where the ${ }^{\wedge}$ means the Hankel transform. This can be extended inductively for every interval $I_{k}(a)$ such that:

$$
\begin{aligned}
& \mu_{f}\left(I_{k}(a)\right)=\sum_{n \in \mathbf{Z}} \mid\left\langle j_{\alpha}\left(z r_{1} \exp \left(2 i \pi \frac{a_{1}}{3}\right)\right) e_{n}\left(z^{3}\right), f\right\rangle \ldots \\
&\left.\left\langle j_{\alpha}\left(z r_{k} \exp \left(2 i \pi \frac{a_{k}}{3^{k}}\right)\right) e_{n}\left(z^{3^{k}}\right), f\right\rangle\right|^{2} \\
&= \exp \left(2 i \pi \alpha \frac{a_{1}}{3}\right) \ldots \exp \left(2 i \pi \alpha \frac{a_{3}}{3^{k}}\right) \sum_{n \in \mathbf{Z}} \mid\left\langle j_{\alpha}\left(z r_{1}\right) e_{n}\left(z^{3}\right), f\right\rangle \ldots \\
&\left.\left\langle j_{\alpha}\left(z r_{k}\right) e_{n}\left(z^{3^{k}}\right), f\right\rangle\right|^{2} .
\end{aligned}
$$

Set $B\left(\left(a_{1}, \ldots, a_{k}\right)\right)=\exp \left(i \pi \alpha a_{1} / 3\right) \ldots \exp \left(i \pi \alpha a_{k} / 3^{k}\right)$ then we get the following:

$$
\begin{aligned}
\mu_{f}\left(I_{k}(a)\right)= & \sum_{n \in \mathbf{Z}} \mid\left\langle j_{\alpha}\left(z r_{1} \exp \left(2 i \pi \frac{a_{1}}{3}\right)\right) e_{n}\left(z^{3}\right), f\right\rangle \\
& \left.\ldots\left\langle j_{\alpha}\left(z r_{k} \exp \left(2 i \pi \frac{a_{k}}{3^{k}}\right)\right) e_{n}\left(z^{3^{k}}\right), f\right\rangle\right|^{2} \\
= & \sum_{n \in \mathbf{Z}}\left|\left\langle j_{\alpha}\left(z r_{1}\right) e_{n}\left(z^{3}\right), f\right\rangle \ldots\left\langle j_{\alpha}\left(z r_{k}\right) e_{n}\left(z^{3^{k}}\right), f\right\rangle\right|^{2} \\
= & B\left(\left(a_{1}, \ldots, a_{k}\right)\right) \sum_{n \in \mathbf{Z}}\left|\prod_{i=1, \ldots, k}\left\langle j_{\alpha}\left(z r_{i}\right) e_{n}\left(z^{3^{i}}\right), f\right\rangle\right|^{2} . \\
= & B\left(\left(a_{1}, \ldots, a_{k}\right)\right) \sum_{n \in \mathbf{Z}}\left|\prod_{i=1, \ldots, k}\left(T_{r_{i}}\left(\bar{f} e_{n}\right)\right) \uparrow\left(3^{i}\right)\right|^{2} .
\end{aligned}
$$

Thus we obtain the desired conclusion. 
Let us now specialize to the case $f=e_{p}$, for some $p \in \mathbf{Z}$. Thus we get

$$
\mu_{p}(.)=\mu_{e_{p}}(.)=\left\|E(.) e_{p}\right\|^{2} .
$$

Set $m_{j}(k)=m_{j}\left(z \exp \left(i 2 \pi \frac{k}{3}\right)\right)=j_{\alpha}\left(r_{j} z \exp \left(i 2 \pi \frac{k}{3}\right)\right)$ where $r_{j}$ are zeros of the Bessel functions $j_{\alpha}$ in the interval $[0,1]$. Then the matrix $m_{j}(k)=m_{j}\left(z \exp \left(i 2 \pi \frac{k}{3}\right)\right)$ with $j, k=0, \ldots, 2$ satisfies the unitarity condition. Hence we have the following result:

Proposition 2. Let $m_{j}(k)=j_{\alpha}\left(r_{j} z \exp \left(i 2 \pi \frac{k}{3}\right)\right)$ be the unitary matrix and let $S_{j}$ be the representation of $\mathrm{O}_{3}$ as in Theorem 1 . Then the measure $\mu_{p}$ is given by the following formula:

$$
\mu_{p}\left(I_{k}(a)\right)=3^{k} \sum_{j \in \mathbf{Z}}\left|\widehat{m_{j}}\left(p-j 3^{k}\right)\right|^{2}, \quad \text { for all } \quad p \in \mathbf{Z}, \quad k \in \mathbf{Z}_{+} \quad \text { and } \quad a \in \Gamma_{3}^{k}
$$

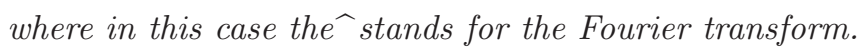

Proposition 3. Let $S_{j}$ be the representation of $O_{3}$ as in Theorem 1 . Let I be the unit interval and let $E($.$) be the corresponding projection-valued measure on B(I)$. Then the induced scalar measure $\mu_{1}()=.\|E(.)\|^{2}$ is the middle-3rd Cantor measure.

Proof. The class of induced measures we are considering were studied in [20]. Every representation of the Cuntz algebra on a Hilbert space $H$ induces a family of measures $\left\{\mu_{f}, f \in H\right\}$. They are projection-valued measures defined on the Borel sets in $X_{3}=\Gamma_{3}^{\mathbf{Z}_{+}}$, where $\Gamma_{3}^{\mathbf{Z}_{+}}=\prod_{\mathrm{z}_{+}} \Gamma_{3}$. In our case we consider them as arising from the particular class of representations of $\mathrm{O}_{3}$ defined in Theorem 1. We observe that in the case that the measure is induced from the permutative representations of the Cuntz algebra the problem of diagonalizing the commutative family of operators

$$
\left\{S_{i_{1}} \ldots S_{i_{k}} S_{i_{l}}^{\star} \ldots S_{i_{1}}^{\star}\right\}
$$

is very simple. The operators monomials from (25) may be realized as multiplication operators on $l^{2}(\mathbf{Z})$. Thus an operator in the family (25) can be represented as multiplication by the indicator function of the set $\sigma_{i_{1}} \circ \sigma_{i_{2}} \ldots \circ \sigma_{i_{k}}(\mathbf{Z}) \subset \mathbf{Z}$ where the maps $\sigma_{i}$ are defined by $\sigma_{i}(x)=\frac{x+i}{3}$. In our case there is a radial translation on the zeros of the Bessel functions. The operators can be realized as multiplication operators by Bessel functions whose argument is on the set $\exp i \pi\left(\sigma_{i_{1}} \circ \sigma_{i_{2}} \ldots \circ \sigma_{i_{k}}(\mathbf{R})\right) \subset \mathbf{C}$. The fact that $\mu_{1}$ is a product measure on $X_{3}$ follows from Prop. 4.1 in [20] since there exists a joint eigenvector $h$ for the operators $S_{i}^{\star}$, i.e., $h=j_{\alpha}\left(r_{n} z\right) 1$, where $i=0, \ldots, 2$, because

$$
S_{k}^{\star}|h\rangle=j_{\alpha}\left(z r_{k} \exp \left(-i 2 \pi\left(\frac{k}{3}\right)\right)\right) \mathbf{1}=\exp \left(-i 2 \pi \alpha\left(\frac{k}{3}\right)\right) j_{\alpha}\left(z r_{k}\right) \mathbf{1} .
$$

Set $\lambda_{i}=\exp \left(-i \pi \alpha\left(\frac{i}{3}\right)\right)$ and let $\omega_{h}()=.\langle h \mid . h\rangle$ be the state corresponding to the vector $h$. This is equivalent to say that the measure is induced from a corresponding 
state $\omega_{h}$ associated to the vector $h$ such that

$$
\omega_{h}\left(S_{i_{1}} \ldots S_{i_{k}} S_{i_{l}}^{\star} \ldots S_{i_{1}}^{\star}\right)=\overline{\lambda_{i_{1}}} \ldots \overline{\lambda_{i_{k}}} \lambda_{i_{l}} \ldots \lambda_{i_{1}}
$$

for all $k, l \in \mathbf{Z}$ and all $i_{1}, \ldots, i_{k} \in \Gamma_{3}$ and $j_{1}, \ldots, j_{l} \in \Gamma_{3}$. Set $p_{k}=p_{a_{1}} \ldots p_{a_{k}}$ to be $B\left(\left(a_{1}, \ldots, a_{k}\right)\right)$. Let $\mu_{1}$ the above Borel measure induced from the state $\omega_{h}$. The support of such a measure can be seen from the following argument to be the middle-3rd Cantor set [20]. In fact

$$
\mu_{1}(A)=\sum_{i=0}^{3-1} p_{k} \mu_{1}\left(\sigma_{k}^{-1}(A)\right) .
$$

Thus the measure $\mu$ satisfies Hutchinson's invariance property so it is a probability measure. Let us observe that the support $X$ of the measure $\mu_{1}$ is the unique solution to

$$
X=\bigcup_{i=0}^{3}\left(\sigma_{i}(X)\right) .
$$

Then $X$ is the middle-3-rd Cantor set on $\mathbf{C}$.

Remark. The Hausdorff dimension of the measure $\mu_{1}$ is

$$
s=\frac{\log (\exp (i \pi \alpha))}{\log 3} .
$$

The measure so constructed is related to the filters of a radial multiresolution in $R^{3}$.

\section{Construction of a fractal measure via radial wavelets}

Let $(X, \mathrm{~B})$ be a measure space and $\sigma$ be a fixed $N$-to- 1 measurable mapping from $X$ to $X$. Let $\sigma: X \rightarrow X$ be onto and B-measurable, i.e., for every $b \in \mathrm{B}, \sigma^{-1}$ (B) $:=$ $\{x \in X \sigma(x) \in \mathrm{B}\} \in \mathrm{B}$. Let $\nu$ be a probability measure defined on B. We assume that the singletons $\{x\}$ for $x \in X$, are in $\mathrm{B}$, but $\nu$ need not be atomic. We also assume that for every $x \in X$, the cardinality of $\sigma^{-1}(\{x)\}$ is $N$, where $N \geq 2$ is fixed and finite. Let $W: X \rightarrow[0, \infty)$ be given and assume that (i) $W$ is measurable, i.e., $W^{-1}(J) \in$ B for every $J$ interval in $[0, \infty)$, (ii) $\sum_{y \in X, \sigma(y)=x} W(y) \leq 1$ for $\nu$ a.e. $x \in X$. The sets $\sigma^{-1}(\{x)\}$ may be labeled by $Z_{N}=\{0,1, . . N-1\}$. Since the singletons are in B, we may pick up measurable branches of the (set-theoretic) inverse $\sigma^{-1}$, i.e., measurable maps $\tau_{i}: X \rightarrow X, i=0,1, \ldots, N-1$ such that $\sigma \circ \tau_{i}=i d_{X}$. Using $W$ we may define a probability of transition or walk from a point $x \in \mathbf{X}$ to one of the points $\tau_{i}(x), i=0, \ldots N-1$, in $\sigma^{-1}(x)$ by

$$
P\left(x, \tau_{i}(x)\right):=W\left(\tau_{i}(x)\right) .
$$

Set $\{\omega(\lambda)\}=\left\{\left(i_{1}, \ldots, i_{n} 0, \ldots, 0\right)\right\}$. Then by iterating we get:

$$
P(x,\{\omega(\lambda)\}):=W\left(\tau_{i_{1}}(x)\right) \ldots W\left(\tau_{i_{n}} \ldots \tau_{i_{1}}(x)\right) .
$$


Below we shall choose $W$ in a particular way so that the random walk will be related to a radial multiresolution. We shall in fact construct a family of probability measure $P_{x}, x \in \mathbf{R}$, on $\mathbf{R}$ induced from the representation of $O_{N}$ given by the operators $S_{i}$ described in Section 2. We prove that it is indeed an infinite convolution measure of Bernoulli type.

For $r, s \in \mathbf{R}$ we consider first the probability measure $\delta_{r} \star_{\alpha} \delta_{s}(f)$ defined on $\mathbf{R}$ by the following:

$$
\delta_{r} \star_{\alpha} \delta_{s}(f):=c_{\alpha} \int_{0}^{\pi} f\left(\sqrt{r^{2}+s^{2}-2 r s \cos \phi}\right) \sin ^{2 \alpha} \phi d \phi,
$$

where $f \in \mathbf{C}_{c}\left(\mathbf{R}_{+}\right)$and $\mathbf{C}_{c}\left(\mathbf{R}_{+}\right)$denotes the space of continuous, compactly supported functions on $\mathbf{R}_{+}$. If we take $f$ to be the normalized Bessel function of order $\alpha$ then we have

$$
\delta_{r} \star_{\alpha} \delta_{s}\left(j_{\alpha}\right)=c_{\alpha} \int_{0}^{\pi}\left(j_{\alpha}\left(\sqrt{r^{2}+s^{2}-2 r s \cos \phi}\right) \sin ^{2 \alpha} \phi d \phi=j_{\alpha}(r) j_{\alpha}(s) .\right.
$$

The functions $r \rightarrow\left(j_{\alpha}(\lambda r)\right) \lambda \in \mathrm{R}_{+}$are exactly those which are bounded and multiplicative with respect to $\star_{\alpha}$. They constitute the dual space of the hypergroup $H_{\alpha}$.

Define the following:

$$
\left\langle\delta_{(r)} \star_{\alpha} \delta_{(s)}, j_{\alpha}\right\rangle=\delta_{(r)} \star_{\alpha} \delta_{(s)}\left(j_{\alpha}\right) .
$$

Then by iteration we get:

$$
\left\langle\delta_{\left(r_{1}\right)} \star_{\alpha} \ldots \star_{\alpha} \delta_{\left(r_{k}\right)}, j_{\alpha}\right\rangle=\delta_{\left(r_{1}\right)} \star_{\alpha} \ldots \star_{\alpha} \delta_{\left(r_{k}\right)}\left(j_{\alpha}\right) .
$$

Let us use the notation: $T_{n_{1}, \ldots, n_{k}}$ to denote $T_{n_{1}} \ldots T_{n_{k}}$. Then by iteration we get:

$$
T_{\tau_{n_{1}}, \ldots, \tau_{n_{k}}} f^{\alpha}(x)=j_{\alpha}\left(\tau_{n_{1}}(x)\right) \ldots j_{\alpha}\left(\tau_{n_{k}} \ldots \tau_{n_{1}}(x)\right) \widehat{f^{\alpha}(x)} .
$$

Thus by (26), (27) we get:

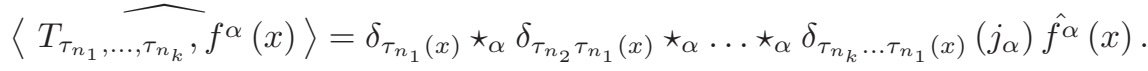

Theorem 4. Let $\sigma: \mathbf{R}_{+} \rightarrow \mathbf{R}+$ and let $\tau_{0}, \ldots \tau_{N-1}$ be a choice of branches of $\sigma$ given by $\tau_{n_{i}}(x)=\exp \left(i 2 \pi \frac{x+n_{i}}{N}\right)$ and $\tau_{n_{k}} \ldots \tau_{n_{1}}(x)=\exp \left(i 2 \pi \frac{x+s}{N^{k}}\right), s=n_{1}+$ $n_{2} N+\cdots+n_{k} N^{k-1}$. Then there exists a measure $P_{x}$ on random walk with the transition probabilities induced from such iterated system on $H_{\alpha}=\left(L^{2}\left(\mathbf{R}_{+}\right), \omega_{\alpha}\right)$ such that

$$
\begin{aligned}
P_{x}(x,\{\omega(x)\}):=W\left(\tau_{i_{1}}(x)\right) \ldots W\left(\tau_{i_{n}} \ldots \tau_{i_{1}}(x)\right)=j_{\alpha}\left(\tau_{i_{1}}(x)\right) & \\
& \ldots j_{\alpha}\left(\tau_{i_{k}} \ldots \tau_{i_{1}}(x)\right),
\end{aligned}
$$

with $W\left(\tau_{i_{j}}(x)\right)=j_{\alpha}\left(\tau_{i_{j}}(x)\right)$. Then it is an infinite Bernoulli convolution measure. 
Proof. We can rewrite the measure $P_{x}$ associated to the random walk defined by the iterated function system $\sigma, \tau_{i}$ as:

$$
P_{x}(x,\{\omega(x)\})=\delta_{\tau_{n_{1}}(x)} \star_{\alpha} \delta_{\tau_{n_{2}} \tau_{n_{1}}(x)} \star_{\alpha} \ldots \star_{\alpha} \delta_{\tau_{n_{k}} \ldots \tau_{n_{1}}(x)}\left(j_{\alpha}\right) .
$$

The above infinite convolution measure, see [9], has distribution function given by

$$
F(x)=\lim _{k \rightarrow \infty} \delta_{\tau_{n_{1}}(x)} \star_{\alpha} \delta_{\tau_{n_{2}} \tau_{n_{1}}(x)} \star_{\alpha} \ldots \star_{\alpha} \delta_{\tau_{n_{k}} \ldots \tau_{n_{1}}(x)}
$$

where the maps $\tau_{i}$ are first defined on $[0,1]$ and extended periodically to $\mathbf{R}_{+}$. The infinite product converges because of the convergence of the series $\sum_{i=1}^{\infty}\left(\frac{1}{N^{2}}\right)^{2}$. The characteristic function is given by the following infinite product $\prod_{i=1}^{\infty} \cos \left(N^{-i} \alpha x\right)$ (see [17]) and it is related to that of a probability measure satisfying Hutchinson's invariance property according to the following lemma.

Lemma 5. Let $\nu_{\lambda}$ be a measure satisfying the following:

$$
\nu_{\lambda}=\left(\nu_{\lambda} \circ \tau_{0}^{-1}+\nu_{\lambda} \circ \tau_{1}^{-1}\right)
$$

where $\tau_{0}(t)=\exp (i 2 \pi(\lambda t+1))$ and $\tau_{1}(t)=\exp (i 2 \pi(\lambda t-1)), \lambda \in(0,1)$. Then the Hankel transform $\widehat{\nu_{\lambda}}$ of $\nu_{\lambda}$ satisfies:

$$
\left.\widehat{\left(\nu_{\lambda}(t)\right.}\right)=\prod_{n=0}^{\infty} \cos \left(2 \pi \alpha \lambda^{n} t\right) .
$$

Hence the measure arising from the above iterated function system is a Bernoulli convolution measure.

Proof. Taking the Hankel transform of $\nu_{\lambda}$ as defined in Lemma 4 we get:

$$
\begin{aligned}
\widehat{\nu_{\lambda}(t)} & =\int j_{\alpha}(x t) d \nu_{\lambda}(x) \\
& =\int j_{\alpha}(\exp (-i 2 \pi(\lambda x-1) t))+j_{\alpha}(\exp (-i 2 \pi(\lambda x+1) t)) d \nu_{\lambda}(x) \\
& =(\cos (2 \pi \alpha t))\left(\widehat{\nu_{\lambda}(\lambda t)}\right) .
\end{aligned}
$$

Then by countable iteration we get:

$$
\widehat{\nu_{\lambda}(t)}=\cos \left(2 \pi \alpha \lambda^{n} t\right)\left(\widehat{\nu_{\lambda}\left(\lambda^{n} t\right)}\right) .
$$

for all $n \in \mathbf{N}$.

Hence in the sense of pointwise limits:

$$
\widehat{\nu_{\lambda}(t)}=\prod_{n=0}^{\infty} \cos \left(2 \pi \alpha \lambda^{n} t\right) \lim _{n \rightarrow \infty}\left(\widehat{\nu_{\lambda}\left(\lambda^{n} t\right)}\right) \text {. }
$$

Thus by the convergence of $\lim _{n \rightarrow \infty}\left(\widehat{\nu_{\lambda}\left(\lambda^{n} t\right)}\right)$ to $\left(\widehat{\nu_{\lambda}(0)}\right)=1$, since $\lambda \in(0,1)$, we obtain that the Hankel transform of the measure $\nu_{\lambda}$ satisfies:

$$
\left.\widehat{\left(\nu_{\lambda}(t)\right.}\right)=\prod_{n=0}^{\infty} \cos \left(2 \pi \alpha \lambda^{n} t\right) .
$$


The measure $\nu_{\lambda}$ arising from the above iterated function system determined by the maps $\tau_{i}$ is a Bernoulli convolution measure, see [24]. Then it follows that the measure on the random walk with transition probabilities given by

$$
P_{x}(x,\{\omega(x)\})
$$

is a Bernoulli convolution measure associated to the iterated function system given by $\tau_{0}$ and $\tau_{1}$. It remains only to remark that it satisfies Hutchinson's invariance property so it is a probability measure.

Lemma 6. Give a fixed $\lambda \in(0,1)$ and assume there is an invariant measure $\mu_{\lambda}$ with respect to the iterated function system as in Theorem 4.

Then the Hankel transform of $\mu_{\lambda}$ satisfies:

$$
\widehat{\mu_{\lambda}(t)}=\prod_{k=0}^{\infty} \exp \left(i 2 \pi \frac{n_{1}+n_{2} \lambda^{-1}+\cdots+n_{k} \lambda^{k-1}}{\lambda^{k}} t \alpha\right) .
$$

Proof. We have

$$
\begin{aligned}
\widehat{\mu_{\lambda}(t)} & =\int_{\mathbf{R}} j_{\alpha}(x t) d \mu_{\lambda}(x) \\
& =\exp \left(\left(i 2 \pi n_{1} \lambda t\right) \alpha\right)\left(\widehat{\mu_{\lambda}(\lambda t)}\right) .
\end{aligned}
$$

Then by iterating:

$$
\widehat{\mu_{\lambda}(t)}=\prod_{n=0}^{\infty} \exp \left(i 2 \pi \frac{n_{1}+n_{2} \lambda^{-1}+\cdots+n_{n} \lambda^{n-1}}{\lambda^{n}} t \alpha\right) \lim _{n \rightarrow \infty}\left(\widehat{\mu_{\lambda}\left(\lambda^{n} t\right)}\right) .
$$

This gives the desired result, since $\left.\lim _{n \rightarrow \infty}\left(\widehat{\mu_{\lambda}\left(\lambda^{n} t\right.}\right)\right)=1$ since $\lambda \in(0,1)$.

\section{Conclusions}

From the sections concerning wavelets constructed in this paper based on products of Bessel functions we conclude that there are a number of interesting question to address such as connections with infinite products measure of Bernoulli type and random walks. Previously studied representations of the Cuntz algebras dependent on a parameter as [25] can be used towards an extension of our considerations to the construction of corresponding random walks on quantum groups.

\section{Acknowledgements}

A. M. Paolucci would like to thank the Max-Planck-Institut für Mathematik in Bonn for the support offered and the excellent working conditions provided during the time this investigation was performed and written. 


\section{References}

[1] S. Albeverio, P. E. T. Jorgensen and A. M. Paolucci, Multiresolution wavelet analysis of integer scale Bessel functions, J. Math. Phys. 48, 073516 (2007) (24 pages).

[2] R. Askey and M. Ismail, Recurrence relations, continued fractions, and orthogonal polynomials, Mem. Amer. Math. Soc. 49, no. 300 (1984).

[3] O. Bratteli, D. E. Evans and P. E. T. Jorgensen, Compactly supported wavelets and representations of the Cuntz relations, Appl. Comput. Harmon. Anal. 8, 166-196 (2000).

[4] O. Bratteli and P. E. T. Jorgensen, Wavelet filters and infinite-dimensional unitary groups, in Wavelet Analysis and Applications (Guangzhou, China, 1999) (D. Deng, D. Huang, R.-Q. Jia, W. Lin, and J. Wang, eds.), AMS/IP Studies in Advanced Mathematics, vol. 25, American Mathematical Society, Providence, International Press, Boston, 2002, pp. 35-65.

[5] O. Bratteli and P. E. T. Jorgensen, Iterated function systems and permutation representations of the Cuntz algebra, Mem. Amer. Math. Soc. 139, no. 663 (1999).

[6] O. Bratteli and P. E. T. Jorgensen, Isometries, shifts, Cuntz algebras and multiresolution wavelet analysis of scale $N$, Integral Equations Operator Theory 28, 382-443 (1997)

[7] V. Chari and A. Pressley, A Guide to Quantum Groups, Cambridge University Press, Cambridge, 1994.

[8] T. S. Chihara, An Introduction to Orthogonal Polynomials, Vol. 13 in Mathematics and its Applications, Gordon and Breach, New York, 1978.

[9] M. Cooper, Dimension, measure and infinite Bernoulli convolutions, Math. Proc. Camb. Phil. Soc. 124, 135-149 (1998).

[10] J. Cuntz, Simple $C^{*}$-algebras generated by isometries, Comm. Math. Phys. 56, 173 185 (1977).

[11] I. Daubechies, Ten Lectures on Wavelets, Vol. 61 in CBMS-NSF. Regional Conf. Ser. in Appl. Math., Society for Industrial and Applied Mathematics, Philadelphia, 1992.

[12] H. Exton, q-hypergeometric functions and applications, Ellis Horwood, Chichester, 1983.

[13] K. Falconer, Fractal Geometry: Mathematical Foundations and Applications, Wiley, Chichester, 1990.

[14] G. Gasper and M. Rahman, Basic Hypergeometric Series, Vol. 35 in Encyclopedia of Mathematics and Its Applications, Cambridge Univ. Press, Cambridge, 1990.

[15] M. Holschneider, Wavelets: An analysis tool, Oxford Science Publications, (1998).

[16] M.E.H. Ismail, The zeros of basic Bessel functions, the functions $J_{\nu+a x}(x)$ and associated orthogonal polynomials, J. Math. Anal. Appl. 86, 1-19 (1982).

[17] B. Jessen, A. Wintner, Distribution functions and the Riemann zeta function, Trans. Amer. Math. Soc. 38, no. 1, (1935).

[18] P. E. T. Jorgensen, Ruelle operators: Functions which are harmonic with respect to a transfer operator, Mem. Amer. Math. Soc. 152, 720, viii+60, (2001).

[19] P. E. T. Jorgensen, Analysis and Probability: Wavelets, Signals, Fractals, SpringerVerlag, Graduate Text in Mathematics, GMT 234, (2006). 
[20] P. E. T. Jorgensen, Measures in wavelet decompositions, Adv. Appl. Math. 23, 561$590(2005)$.

[21] P.E. T. Jorgensen, K. Kornelson and K. Shuman, Orthogonal exponentials for Bernoulli iterated function systems, arXiv:math/073385v1, (2007).

[22] P. E. T. Jorgensen, L. M. Schmitt, and R. F. Werner, $q$-canonical commutation relations and stability of the Cuntz algebra, Pacific J. Math. 165, 131-151 (1994).

[23] P. E. T. Jorgensen and R. F. Werner, Coherent states of the $q$-canonical commutation relations, Comm. Math. Phys. 164, 455-471 (1994).

[24] P. E. T. Jorgensen, Measures in wavelet decompositions, Advances in Applied Mathematics, 34, issue 3, 561-590 (2005).

[25] P. E. T. Jorgensen, A. M. Paolucci, Wavelets in mathematical physics: $q$-oscillators, J. Phys. A: Math. Gen. 36, 6483-6494 (13 June 2003).

[26] K. Knopp, Theory and application of infinite series (Blackie, 1951) (translation of Theorie und Anwendung der unendlichen Reihen, 1941).

[27] S. G. Mallat, Multiresolution approximations and wavelet orthonormal bases of $L^{2}(\mathbf{R})$, Trans. Amer. Math. Soc. 315, 69-87 (1989).

[28] A. M. Paolucci, On a $C^{*}$-algebra based on the Hahn-Exton $q$-Bessel functions, Bessel Functions and Cuntz Algebras Representations, Aplimat 2007, Part II, pp. 109-114 (2007).

[29] A.D. Poularikas, ed., The Transforms and Applications Handbook, The Electrical Engineering Handbook Series, CRC Press, Boca Raton, FL; IEEE Press, New York, 1996.

[30] H. Rauhut, M. Rösler, Radial multiresolution in dimension three, Constr. Approx. 22, 193-218 (2005).

[31] I. N. Sneddon, Fourier Transforms, McGraw-Hill, New York, 1951; Dover, New York, 1995.

[32] K. Trimeche, Generalized Wavelets and Hypergroups, New York, Gordon and Breach, 1997.

[33] G. N. Watson, A Treatise on the Theory of Bessel Functions, second edition, Cambridge University Press, Cambridge; The Macmillan Company, New York, 1944; reprinted, Cambridge Mathematical Library, Cambridge University Press, Cambridge, 1995.

Sergio Albeverio

Institut für Angewandte Mathematik

Universität Bonn

Wegelerstr. 6

D-53115 Bonn

Germany

and

SFB 611, Bonn, BiBos (Bielefeld-Bonn)

IZKS Bonn

CERFIM (Locarno) and ACC. ARCH (USI)

Switzerland

e-mail: albeverio@uni-bonn.de 
Anna Maria Paolucci

Max-Planck-Institut für Mathematik

Vivatsgasse 7

D-53111 Bonn

Germany

e-mail: paolucci@mpim-bonn.mpg.de

Communicated by Palle Jorgensen.

Submitted: February 20, 2008.

Accepted: March 6, 2008. 